\title{
A New Rotational Stepwise Mechanical Energy Harvester for Biomedical Implants
}

\author{
Nabiollah Shiri, ${ }^{1 *}$ Hadi Veladi, ${ }^{1}$ and Hanieh Niroomand Oscuii ${ }^{2}$ \\ ${ }^{1}$ Microsystem Fabrication Lab, University of Tabriz, Tabriz 51666-1581, Iran \\ ${ }^{2}$ Mechanical Engineering Faculty, Sahand University of Technology, Tabriz 51335-1996, Iran \\ (Received September 11, 2017; accepted November 30, 2017)
}

Keywords: mechanical energy harvester, biomedical implants, actuator, storage, ratchet

A new mechanical energy harvester (EH) has been proposed, which can harvest, store, and deliver energy in mechanical form. This harvester can be exploited in bioimplantable devices. The structure composed of three individual parts, namely, the actuator, storage, and latch, accumulates mechanical energy in a conventional spiral spring in terms of stepwise rotation. A ratchet mechanism latches the spring and releases it when energy delivery is required. Various cells are defined and their performances are compared under sequential mechanical forces of up to $7 \mathrm{~N}$ amplitude, which models heart vein vibration force. The storage part will store $3.21 \mathrm{~mJ}$ energy in a single-step rotation. The total area of the structure was $30.06 \mathrm{~mm}^{2}$ with a thickness of $0.7 \mathrm{~mm}$. The proposed mechanical microstructure shows an effective solution in bioimplantable EHs.

\section{Introduction}

With the rapid development of micro-electromechanical systems (MEMS) and lowpower implantable medical devices, efficient power supply has become a crucial engineering challenge. Over the last few years, there has been growing interest in energy-harvesting devices to allow autonomous operation in the control and monitoring of energy supply. Consequently, these systems do not require any power supply from the grid. Energy harvesters (EHs) create no carbon emission and are therefore environmentally friendly. They are also more economical than traditional systems, which usually require battery replacement or frequent recharging. Energy harvesting from the ambient has become possible over the last few decades. ${ }^{(1,2)}$ EHs typically convert a small amount of environmental energy, which is usually wasted, into electrical energy on the order of $\mu \mathrm{W}$ to $\mathrm{mW}^{(3)}$ An EH acts as a rechargeable battery in order to guarantee a stable supply to the load. For some specific applications, the harvester itself can supply power when required in the absence of a battery. ${ }^{(4)}$ Available energy sources in the environment could be in the form of vibration, ${ }^{(5-9)}$ light, ${ }^{(10,11)}$ heat, ${ }^{(12-14)}$ hydraulic, ${ }^{(4,15)}$ pressure, ${ }^{(16-18)}$ force, ${ }^{(19,20)}$ and so forth. For an implantable EH, different vibrating organs are attractive energy sources owing to the unlimited operation time and distributed locations

*Corresponding author: e-mail: nabi.shiri@tabrizu.ac.ir

http://dx.doi.org/10.18494/SAM.2018.1740 
around the body. In kinetic EHs or vibration power generators, ${ }^{(6)}$ mechanical energy sources have different excitation frequencies: less than $10 \mathrm{~Hz}$ for human movements and over $30 \mathrm{~Hz}$ for machinery vibrations. ${ }^{(21)}$ Adaptive kinetic EHs are developed to increase the operational frequency range of vibration EHs, thus addressing the bandwidth limitation. ${ }^{(22)}$

The most common technologies for absorbing vibration or kinetic energy include piezoelectric, ${ }^{(5,23,24)}$ electrostatic, ${ }^{(25,26)}$ and electromagnetic ${ }^{(19,27,28)}$ conversion. While piezoelectric, conversion can provide adequate levels of voltage, low power supplement, and toxicity of materials with high piezoelectric coefficients together with their inherent brittle structure make them less appropriate for implantable devices. The electrostatic conversion as an alternative method requires an isolated voltage source as the reference potential. On the other hand, the electromagnetic conversion offers high power efficiency but provides low resonant frequency due to a rather bulky ferromagnetic core. However, the device generates low-level output voltage. ${ }^{(28)}$ The energy levels obtained using these techniques are fairly small to supply demanding applications such as data transmission, sensor nodes, and implantable medical devices. Therefore, to design an implantable EH, the capacity of the source, its intensity, biocompatibility, implantable feasibility, and cost analysis are the main criteria. By considering these limitations and the efficient operation of implantable $\mathrm{EH}$, a great challenge will be encountered.

In a typical $\mathrm{EH}$, normally a transducer is necessary to convert the available environmental energy into electrical form, which is more convenient to be stored. ${ }^{(4)}$ Moreover, an energy management is required, which is an interface element either to drive the consumption device or to control the storing function. ${ }^{(4)}$ An integrated $\mathrm{EH}$ could be a mainstream requirement to produce an easy-to-implant, lighter, cheaper, and therefore more suitable $\mathrm{EH}$ for mobile or remote applications. To achieve this, eliminating a bulky transducer element seems to be an essential step. Mechanical EHs are more convenient devices owing to the availability of resources when compared with the others regarding implantable devices.

In this research, a new all-mechanical EH is examined. Mechanical energy in the form of heartbeat vibrations is stored in mechanical form with no requirement of conversion to electrical or other forms of energy. In Sect. 2, the idea of a new mechanical EH is expressed, and different parts of the proposed EH, namely, actuator, stepper spring cell (storage), and ratchet will be introduced. All parts of the proposed EH are evaluated using a mathematical model and equations to confirm its proper operation. Section 3 shows simulation results of the EH. Also, the advantages and disadvantages of the structure will be discussed. All designs and analyses carried out are similar to satisfy medical implantation device requirements and fabrication feasibility.

\section{Proposed Energy-harvesting Cell}

Inside the human body, there are abundant sources of mechanical energy, especially vibration. For instance, heartbeat produces $0.5 \mathrm{~J} / \mathrm{s}$. This could be a significant source of power especially for implantable pacemakers. Some piezoelectric devices have already been studied to harvest this energy. ${ }^{(29)}$ The size of the human heart in an adult person is about $6 \times 12 \times 9$ $\mathrm{cm}^{3}$, with a mean volume of about $648 \mathrm{~cm}^{3}$. According to these dimensions, a mechanical cell 
is proposed, which can harvest vibration energy from heart muscles or main heart vessels. We focused on the all-mechanical EH to eliminate transduction, which as explained is more suitable for implantable devices.

Inspired from automatic watches, the storage cell is a spiral spring encountered with a geared peripheral to assist power transfer. Figure 1 shows an energy storage cell (primary storage cell) structure. The spring is a spiral with a rectangular cross section anchored to the center of the rotating ring and supports the ring on a peripheral link. The sawtooth around the ring can also be considered as mechanical energy sensors, which are sensing energy at different points, and by engaging the drive components, transfer energy to the spiral (storage) element. The storage cell is loaded by a torque in the direction of the winding. The torque originating from the actuator is small and the created angular deflection and the stored energy could be calculated as follows.

From different storage parameters specified in Fig. 1, the angular deflection of the cell, $\theta$ (in degree), resulted as Eq. (1).

$$
\theta=\frac{2160 M L}{\pi E b t^{2}}
$$

Here, $M$ is actuating torque, $E$ is modulus of elasticity, $b$ is the width of the spring strip, $t$ is the thickness of the cell, and $L$ is the spring mean length, which is determined using Eq. (2).

$$
L=n \pi\left(R_{i}+R_{o}\right)
$$

Here, $n$ represents the number of windings, and $R_{i}$ and $R_{o}$ are inner and outer radii, respectively. The bending stress of the spring is obtained using Eq. (3) and used to estimate the stored energy.

$$
\sigma=K_{b} \frac{6 M}{b t^{2}}
$$

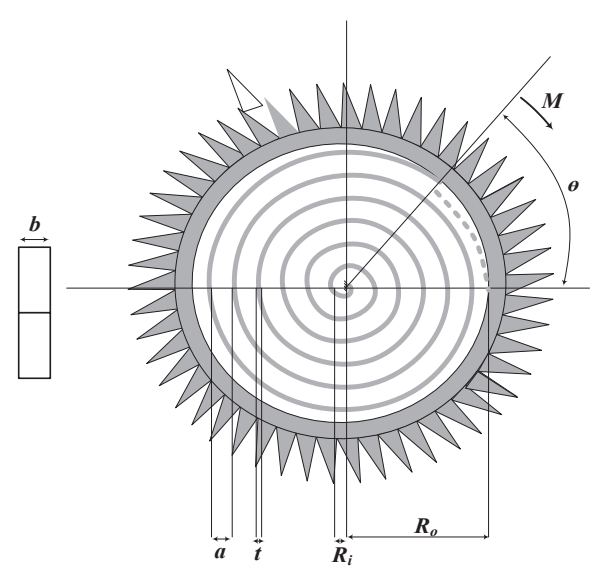

Fig. 1. Energy storage cell. 
Here, $1<K_{b}<2$ is the curvature correction factor and represents the additional stresses resulting from spiral curvature. Equations (1)-(3) are sufficiently precise in low loading torques, linear working characteristics, and low spring constant. The number of sawtooth elements and spiral swirl are the main parameters of the storing cell, in the selection of the appropriate cell under given conditions.

The actuation is delivered to the storage element using a designated mechanism as shown in Fig. 2. This in fact is a simple compliant structure designed to either transfer energy to the storage by forced rotation or to release it to the load when required.

The actuating part is supported only on one anchor (point $\mathrm{D}$ ) and absorbs mechanical energy in terms of torques on $\mathrm{AB}$ or $\mathrm{BC}$ beams. If the torque applies over the $\mathrm{AB}$ segment with $\mathrm{B}$ as the pivot, the tip E moves downward and causes the storage cell to rotate counterclockwise. On the other hand, with torques applied on BC segment, the tip moves upward and forces the cell to rotate clockwise. Therefore, on the basis of the mutual direction of the sawtooth on the drive and storage elements (the arrow-shaped tip and tooth), the cell will store (charge) or deliver (discharge) mechanical energy.

The structural analysis of the actuator for the accurate calculation of elastic rotation $(\delta)$ and rotation angle $(\theta)$ at point $\mathrm{E}$ is very complicated but it is possible to obtain by some reasonable approximations. By neglecting shear stress and axial force effects, the bending moment of each part will be calculated. Also, by using the virtual vertical force $Q$ in point E, the bending moment of each part will be calculated. Finally, by using the principle of superposition and Castigliano's theorem deflection of the structure, $\left(\delta_{\max }\right)$ is calculated as Eqs. (4-1)-(4-4). Considering uniform thickness $(t)$ for all parts of the structure, the dimensions are described in Fig. 2.

$$
\delta_{\max }=\int_{0}^{L_{4}} \frac{M}{E I} \frac{\partial M}{\partial Q} d x
$$

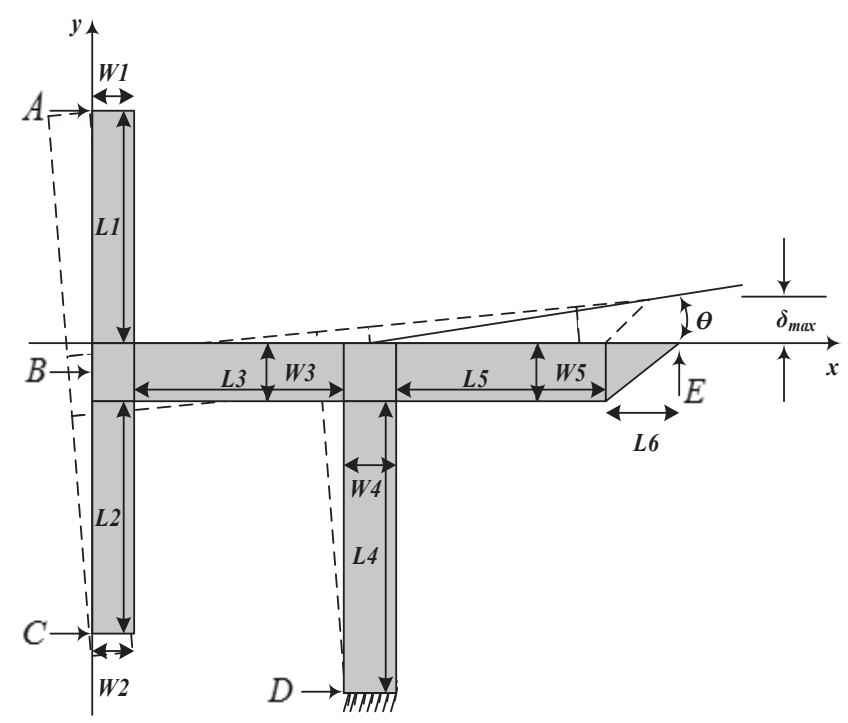

Fig. 2. Mechanical actuator for the structure. 


$$
\begin{gathered}
\delta_{\max }=\int_{0}^{L_{4}} \frac{Q\left(L_{5}+L_{6}\right)+F L_{2}+F x}{E I}\left(L_{5}+L_{6}\right) d x \\
\delta_{\max }=\frac{L_{5}+L_{6}}{E I}\left\{Q\left(L_{5}+L_{6}\right) x+F L_{2} x+\frac{F x^{2}}{2}\right\}_{0}^{L_{4}} \mid Q=0 \\
\delta_{\text {max }}=\frac{L_{5}+L_{6}}{E I}\left(F L_{2} L_{4}+\frac{F L_{4}^{2}}{2}\right)
\end{gathered}
$$

The rotation angle $(\theta)$ at point $\mathrm{E}$ is calculated using Eq. (4-4), which is divided by the effective length of the structure and shown by Eq. (5); here, $\theta$ is small enough to use tangent approximation.

$$
\tan \theta=\frac{\delta_{\max }}{L_{5}+L_{6}} \Rightarrow \theta=\frac{\delta_{\max }}{L_{5}+L_{6}}
$$

The structural analysis of the actuator in Eqs. (4-4) and (5) shows that the most important parameters affecting the rotational behavior of the actuator at point $\mathrm{E}$ are $L_{4}$ and $W_{4}$. As a second degree of sensitivity, $L_{2}, L_{5}, L_{6}$, and $t$ are important. $L_{3}$ has a negligible effect at point E's deflection, while it has a significant effect on the tensile strength of the actuator during charging and discharging. It is worth noting that $L_{1}$ has virtually no effect on discharging. $L_{2}$ has a clearly opposite role to that of $L_{1}$.

To latch the spring after each rotation and prevent unwanted discharge, a ratchet structure has been included as shown in Fig. 3. The ratchet has once again been designed with an arrow-shaped tip to assist the engagement to the gears. All tips are made as a right triangle to enable driving in one direction while latching in the other direction of rotation. The ratchet is supported in one end (flat end) and is actuated by a torque in the other end (triangular end). By approximating the tip of the ratchet to a quadrilateral surface, the ratchet behaves like a cantilever beam actuated with force $(F)$ at the free end.

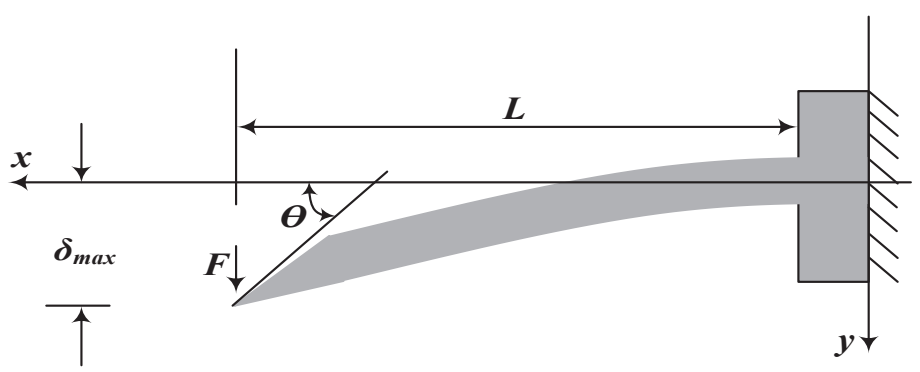

Fig. 3. Ratchet. 
The rotation angle of the free end of the ratchet $(\theta)$ could be estimated using Eq. (6).

$$
\theta=\frac{F L^{2}}{2 E I}
$$

Here, $F$ is the actuating force that resulted from rotation, $E$ is the modulus of elasticity, $L$ is the length of the ratchet beam, and $I$ is the area moment of inertia of the ratchet's cross section. Mathematical analysis of the ratchet gives the maximum elastic deflection of the ratchet $\left(\delta_{\max }\right)$ as Eq. (7).

$$
\delta_{\max }=\frac{F L^{3}}{3 E I}
$$

The parameters of Eqs. (6) and (7) are derived from Eq. (1).

The overall energy-harvesting cell is shown in Fig. 4, which consists of three parts: the actuator, storage, and latch. Any mechanical force obtained from the ambient absorbs as a torque to the pivot $\mathrm{B}$. This could be presented by a coupling force to points $\mathrm{A}$ and $\mathrm{C}$ and leads to counterclockwise or clockwise rotation with respect to the pivot. Any clockwise rotation would be absorbed by a storage element as described later, while the counterclockwise excitation only results in disengagement. For example, if a mechanical force actuates a point between $\mathrm{B}$ and $\mathrm{C}$, the actuator drives the storage part to rotate clockwise, and if the input actuating torque is sufficiently high to rotate the storage part about one step, the holder part will lock into the next available tooth of the storage part. When latched, the holder together with the actuator prevents any more ring rotation until the next driving step arrives. The right triangle shape of the tips both in holders and around the ring facilitates this type of asymmetric engagement.

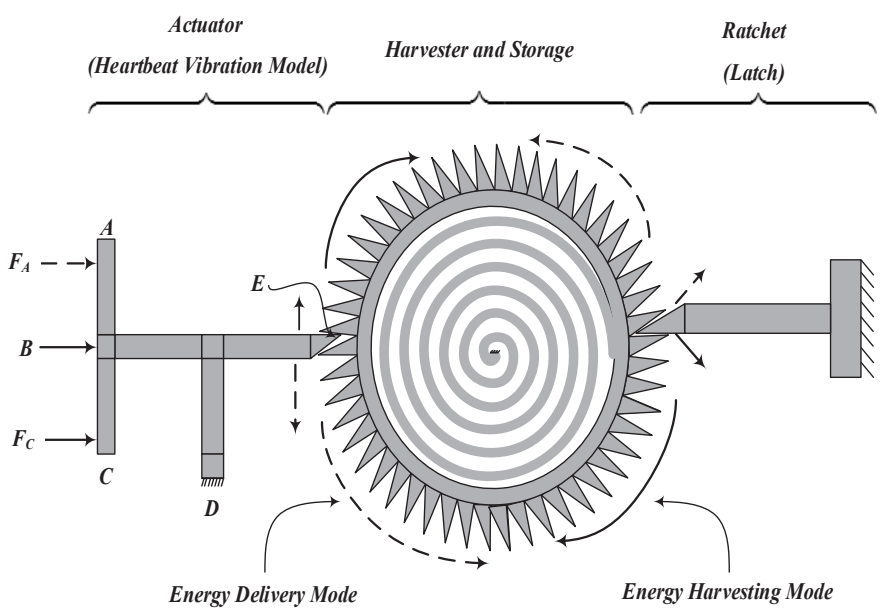

Fig. 4. Total-energy cell; harvesting (solid line) and delivering (dashed line) energy procedures. 
Figure 4 shows energy-harvesting and energy-delivering operation modes of the proposed structure with solid and dashed lines, respectively. When an actuating force $\left(F_{C}\right)$ is applied to the actuator, its tip moves upward, which causes the storage part to rotate clockwise. Then the ratchet bends downward and finally is locked into the next tooth. At this state, mechanical energy will be stored in the cell in the form of mechanical stress. On the other hand, a mechanical-energy-delivering procedure starts as a small force $\left(F_{A}\right)$ applied to the mechanical actuator, which causes the storage part to rotate counterclockwise, and the ratchet bends upward and the mechanical stress, which has been stored in the storage part, will cause the storage part to rotate. Note that $F_{A}$ is much smaller than $F_{C}$, which results in a smaller release force.

\section{Simulation Results and Discussion}

The proposed cell has been assessed for potential biomedical applications. Here, the actuating force $F_{C}$ can be any vibration force produced by an organ inside the body, e.g., heartbeat or heart vessels. In practice, the latter provides a more acceptable and a more available source of power to be harvested. The heart pumps blood through the body, normally at the rate of 72 beats per minute for a healthy adult. Arterial blood pressure ranges from 120 $\mathrm{mmHg}\left(=15789 \mathrm{~Pa}=15789 \mathrm{~N} / \mathrm{m}^{2}\right.$ ) (systolic) to $80 \mathrm{mmHg}$ (diastolic). A heart's systolic pressure produces a force that has been considered and modeled as a mechanical actuating force $\left(F_{C}\right)$. For the aorta with a mean diameter of about $2.5 \mathrm{~cm}\left[A=\pi(2.5 / 2)^{2}=4.91 \times 10^{-4} \mathrm{~m}^{2}\right]$, Eq. (8) obtains the amount of actuating force as

$$
P=\frac{F}{A} \Rightarrow F=P A=15789 \times 4.91 \times 10^{-4} \Rightarrow F=7.75(\mathrm{~N}) .
$$

The force of $7 \mathrm{~N}$ has been considered to supply the harvester. A simulation of the proposed energy-harvesting cell has been performed to assess the storage and latch behavior.

\subsection{Material effects}

To study material effects, the actuator was designed using three alternative materials: silicon (Si), stainless steel type 304 (SS), and plexiglass [poly(methyl methacrylate), PMMA]. All devices have similar lengths and widths but different thicknesses. The latter is varied to obtain comparable results for all devices. Table 1 shows material properties and dimensions for $\mathrm{Si}, \mathrm{SS}$, and plexiglass.

Table 1

$\mathrm{Si}, \mathrm{SS}$, and plexiglass actuator dimensions.

\begin{tabular}{lccc}
\hline & $\mathrm{Si}$ & $\mathrm{SS}$ & Plexiglass \\
\hline Young's modulus $(\mathrm{GPa})$ & 150 & 195 & 3.3 \\
Yield stress $(\mathrm{GPa})$ & 5 & 0.215 & 0.11 \\
Thickness $(\mathrm{mm})$ & 0.7 & 2 & 28 \\
Total length $(\mathrm{mm})$ & 6.26 & 6.26 & 6.26 \\
Total width $\left(\mathrm{mm}^{2}\right)$ & 5 & 5 & 5 \\
\hline
\end{tabular}


Using these dimensions, the actuator was simulated and the displacements in the $X, Y$, and $Z$ directions were evaluated. While a range of forces in 1 to $8 \mathrm{~N}$ stimulates the actuators, a $0.1 \mathrm{~N}$ load was applied at point $\mathrm{E}$ as a load to simulate the rest of the cell. The actuator's operation is then studied to obtain the maximum allowable deflection at the tip (E) far below the structure's fracture damage.

Figure 5 shows the maximum deflection of actuators made by different materials versus applied forces. Si and plexiglass have very similar behaviors (remember plexiglass has a thickness of about 30-fold), while their deflections are much more than those of SS. This arises from the fact that we considered SS as thicker than Si (and therefore, the SS actuator is stiffer than the Si one) to be able to cope with its small yield stress as shown in Table 1.

It is resulted from Fig. 5, that is, for given dimensions, Si presents better mechanical behavior in a smaller cross section.

\subsection{Actuator results}

The proposed actuator has been analyzed to find the points with stress concentration and to achieve the optimal design with maximum deflection and maximum allowable stress as shown in Fig. 6.

The actuator receives $7 \mathrm{~N}$ force (which corresponds to the heart vessels' vibration forces), which causes stresses in different parts of the actuator, which is safely less than the Si's yield stress (5-9 GPa). ${ }^{(30)}$ Simulation results for optimum dimensions confirm proper operation with the least off-plane deformation.

To evaluate the model, the deflection of point E resulting from Eq. (4-4) is compared with the simulation results and shown in Fig. 7. The diagram shows a significant difference between the simulation and equation results although the patterns are quite similar. This difference arises from the approximations in Eq. (4-4). This is in contrast to a more precise nonlinear analysis for large deflections, which were used during finite element method (FEM) analysis

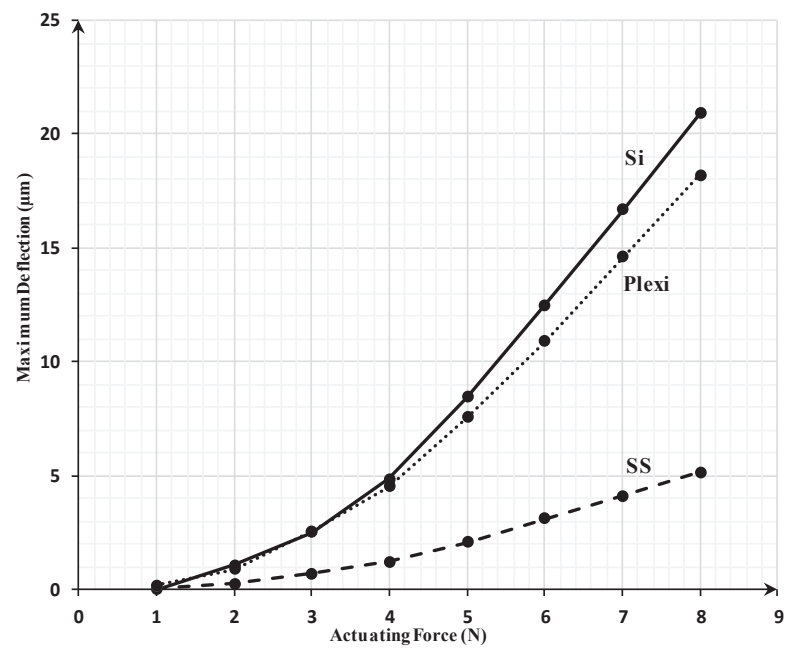

Fig. 5. Maximum deflection of actuator. 

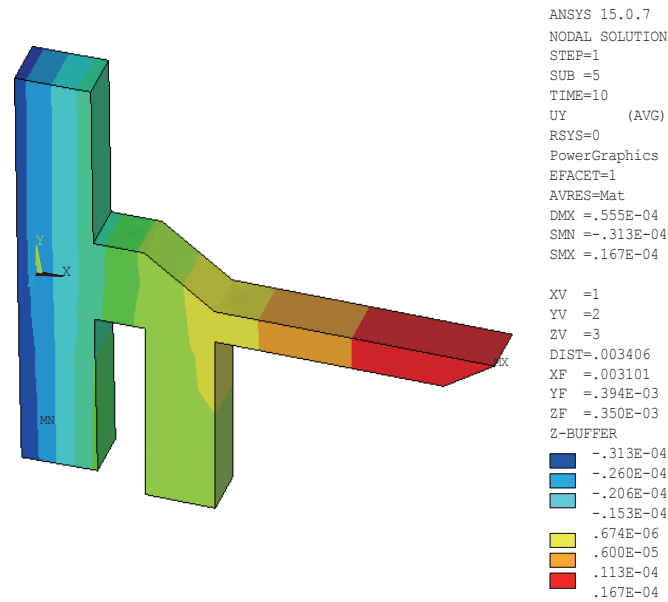

Fig. 6. (Color online) Deflection of actuator structure $(\mathrm{Si})$.

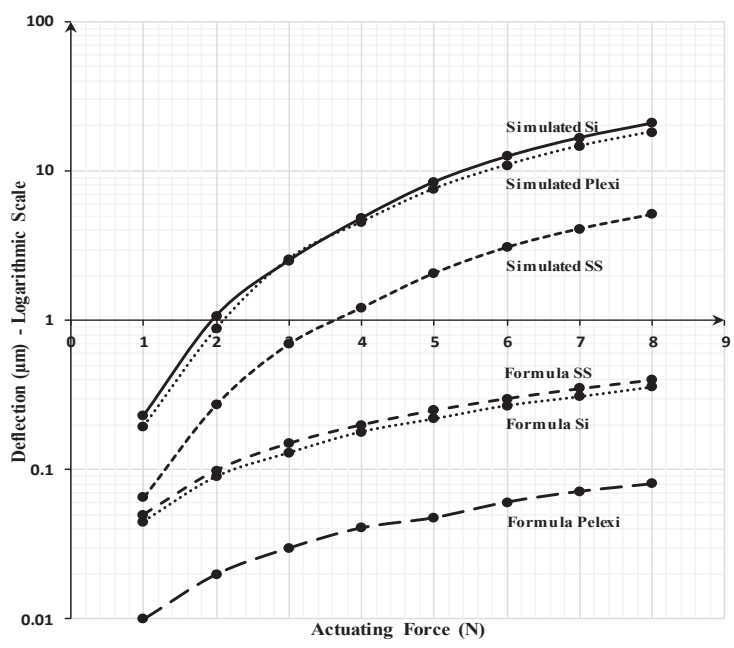

Fig. 7. Comparison of simulation results with those from formula.

and could explain the meaningful difference shown in Fig. 7. Nevertheless, we believe that the similarity could confirm the FEM analysis to certain degrees.

\subsection{Storage results}

With $\mathrm{Si}$ as the selected material for the cell, the spiral and the associated gear were also assessed to ensure efficient storage. The main parameters of interest to design the storage element are thickness, the number of spiral turns, and its width, which affect its stiffness and therefore absorbed energy. The actuator's tip movement, which is applied to the storage, is calculated and modeled as a force proportional to the actuating force. For example, if $F_{C}$ is set with $7 \mathrm{~N}$, then the equivalent moment for the storage is $0.018 \mathrm{Nm}$. To model the ratchet effect on the storage, the storage is loaded with $0.001 \mathrm{Nm}$ moment at a $180^{\circ}$ distance from the actuation point. Figure 8 shows a stress contour map for a typical storage with 5 turns and 58 teeth gear under $0.018 \mathrm{Nm}$ actuation moments, which is related to $7 \mathrm{~N}$ as $F_{C}$.

Equivalent actuating moments were applied to a typical Si storage, and the related rotation angle of the storage was measured and compared with the Eq. (1) results, as shown in Fig. 9. The resulting rotation angle in Fig. 9 is for the Fig. 8 storage with a $0.7 \mathrm{~mm}$ thickness, 5 turns of the spiral, the spiral width of $0.2 \mathrm{~mm}, 58$ teeth, and a total area of $45.4 \mathrm{~mm}^{2}$.

Figure 9 shows that the rotation angles of storage for the simulated storage and its formula [Eq. (1)] show similar behaviors, and the difference arises from formula approximation; thus, Eq. (1) could be improved using a coefficient.

Another scheme to study the cell's behavior is in terms of the absorbed mechanical energy. Since elastic energy is proportional to the area under the stress-strain curve (i.e., $E_{e}=1 / 2 \int \sigma$. $d \varepsilon=1 / 2 E \int_{\sigma \cdot d \sigma}=\sigma^{2} / 2 E$ ), with $E_{e}, \sigma, \varepsilon$, and $E$ as elastic energy, stress, strain, and Young's modulus, respectively, a larger stress in a rather stiff material such as $\mathrm{Si}$ with $E=130-188 \mathrm{GPa}$ results in a larger absorbed energy (note that energy is proportional to $\sigma^{2}$ but reversely to $E$ ). 

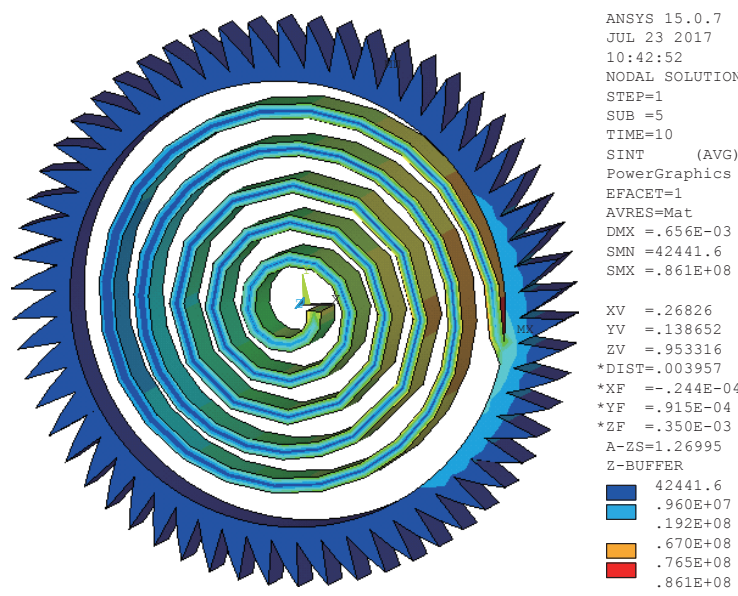

Fig. 8. (Color online) Typical storage stress contour map.

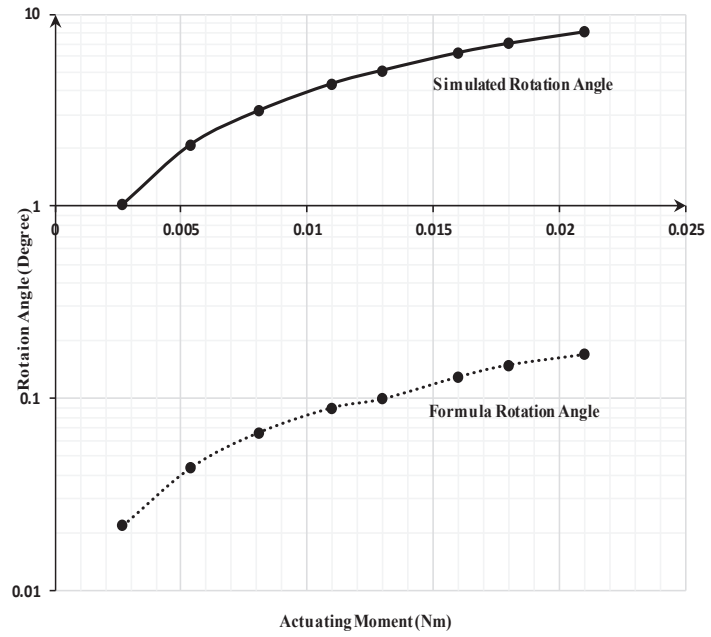

Fig. 9. Rotation angle of storage from simulated storage and its formula.

Therefore, it is important to derive stress variations along the cell to both estimate the absorbed energy and determine stress concentration points. The latter helps to obtain an optimum design for given constraints. Figure 8 shows stress intensity maps for a typical cell with 58 teeth under the actuation force of $7 \mathrm{~N}$. The obtained stress contour shows that the maximum stress is about 86.1 MPa. This stress is concentrated on the engaged teeth and also on the spiral-ring joint. The absorbed energy is distributed along the spiral as deduced from the map.

To be able to compare cells with parameter variations, results of simulation for the different Si device structures as listed in Table 2 have been obtained. Initially, the spirals with 2 turns under load conditions similar to Fig. 8 are considered, and the effects of ring width and the number of teeth are studied for varying thicknesses of the storage cell. It is worth mentioning that the columns of Table 2 show equivalent cells, which means that it is possible to maintain the mechanical behavior of the cell while achieving finer steps (larger number of teeth) by maintaining the spiral structure but increasing the cell's thickness.

In Table 2, equivalent cells have the same features in terms of the number of spiral turns and width, but the thickness and number of teeth are different. Cells 1, 2, 3, and 4 are equal; it means that if an actuating force of $7 \mathrm{~N}$ is applied, then all of the mentioned cells will rotate about one tooth. On the other hand, a cell 1 structure with $N=58$ teeth around has $\theta=360 / 58$ $=6.2$ degrees of rotation per step. This is about $\theta=360 / 10=36$ degrees for cell 4 with 10 teeth around. Thus, different cells with different angles of rotation could be extracted. Alternatively, one may study the effects of spiral turns on storage efficiency.

\subsection{Ratchet results}

Next, the mechanical behavior of the ratchet was assessed. The actuating force applied to the actuator causes the rotation of the storage. This rotation is applied to the ratchet and would deflect it. The force on the ratchet can be calculated on its tip for a known rotation angle of the storage, which itself is driven by an actuating force of 1 to $8 \mathrm{~N}$. 
Table 2

Equivalent cells with different numbers of sawteeth and different thicknesses.

\begin{tabular}{llllll}
\hline & Cell 1 & Cell 2 & Cell 3 & Cell 4 \\
\hline Thickness (mm) & 0.7 & 0.6 & 0.5 & 0.4 \\
\hline Number of spiral turns & 2 & 2 & 2 & 2 \\
\hline \multirow{3}{*}{ Spiral ring width (mm) } & 0.15 & 0.15 & 0.5 & 0.5 \\
& 0.12 & 0.12 & 0.12 & 0.12 \\
& 1 & 1 & 1 & 1 \\
Number of sawteeth & 58 & 48 & 38 & 32 \\
& 28 & 24 & 20 & 16 \\
Rotation (sawtooth) & 18 & 1 & 1 & 1 \\
\hline & & & & &
\end{tabular}

Fig. 10. Ratchet deflection.

Figure 10 shows the deflection of the tip vs the actuating force. Simulation results are compared with those of Eq. (7). At a small deflection, the simulation and formula have similar results, but at a large deflection, the curves will have a greater difference. This emphasizes that more accurate results come from the FEM analysis, which deploys a large deflection modeling.

The safe operation of the ratchet can be examined by the analysis of the stress on the ratchet as depicted in Fig. 11. The ratchet has $0.7 \mathrm{~mm}$ thickness, $1.2 \mathrm{~mm}$ length (beam length is 1 $\mathrm{mm}$ ), and $0.2 \mathrm{~mm}$ width. A force of $7 \mathrm{~N}$ is applied to the tip, and as expected, the maximum stress occurs at the support, which is $2.27 \mathrm{GPa}$. This clearly presents a normal beam bending behavior.

\subsection{Total cell results}

Finally, all parts of the cell were studied while coupled together, and the cell energy harvesting operation was closely examined. As a coupled system, the actuator harvests heart vessels' vibration energy and stores the energy in the spiral storage in terms of elastic energy. The ratchet locks the cell against unwanted discharging. On the basis of the actuator, storage, and ratchet dimension for the Si material, a typical final cell was designed. The dimensions of the cell are summarized in Table 3.

The typical cell has 78 teeth and two spirals; the total area is $30.06 \mathrm{~mm}^{2}$ and its total volume is $23.142 \mathrm{~mm}^{3}$. The cell is loaded with $7 \mathrm{~N}$ force at the actuator, and the total deflection of the cell is shown in Fig. 12.

A range of 1 to $8 \mathrm{~N}$ forces are applied to the actuator in Fig. 12, and the mechanical stress of the cell in spiral parts was measured and shown in Fig. 13.

As the actuating force increases, the stored energy in the form of stress will increase. A more accurate analysis of the cell is carried out by considering the effects of the number of spirals and the spiral width on the stored energy. With a $7 \mathrm{~N}$ force applied to the actuator and then an equivalent force applied to the storage, Fig. 14 shows how changing the spiral number and its width affect the stored energy. The three curves in Fig. 14 show stored energy in the proposed cell with 1 (solid line), 2 (dotted line), and 5 (dashed line) turns of spirals while the spiral width is changing. 


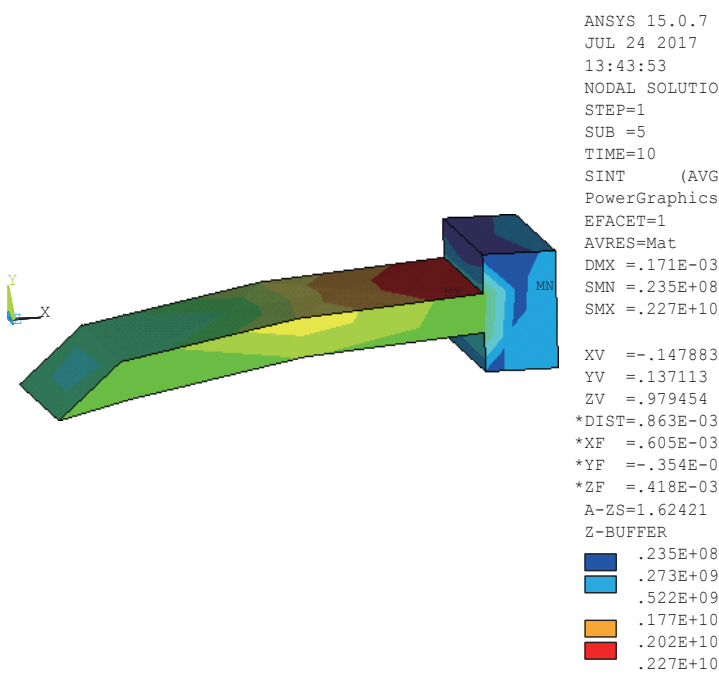

Fig. 11. (Color online) Ratchet stress.

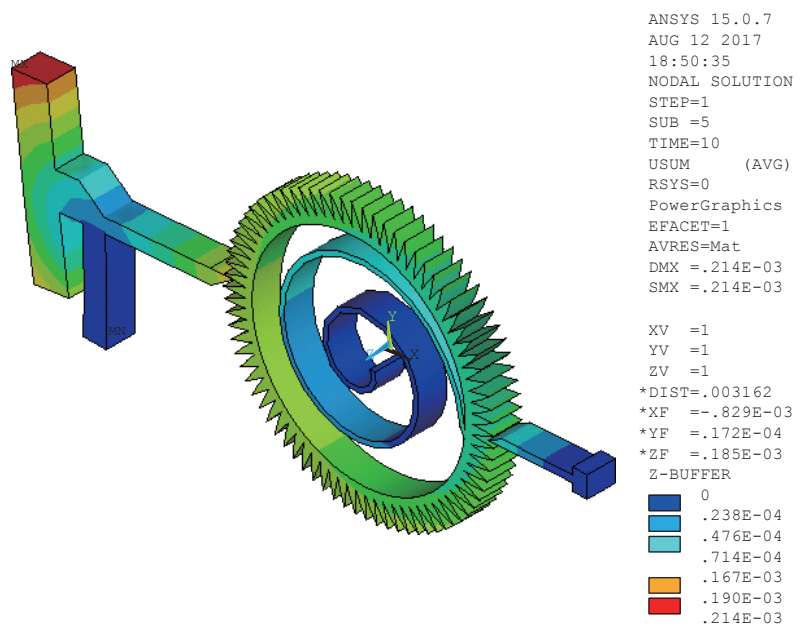

Fig. 12. (Color online) Deflection of total cell.
Table 3

Dimensions of energy-harvesting parts.

\begin{tabular}{lccc}
\hline & Actuator & Storage & Ratchet \\
\hline Thickness $(\mathrm{mm})$ & 0.7 & 0.7 & 0.7 \\
Area $\left(\mathrm{mm}^{2}\right)$ & 8.75 & 14.44 & 0.28 \\
Volume $\left(\mathrm{mm}^{3}\right)$ & 6.125 & 10.108 & 0.196 \\
\hline
\end{tabular}

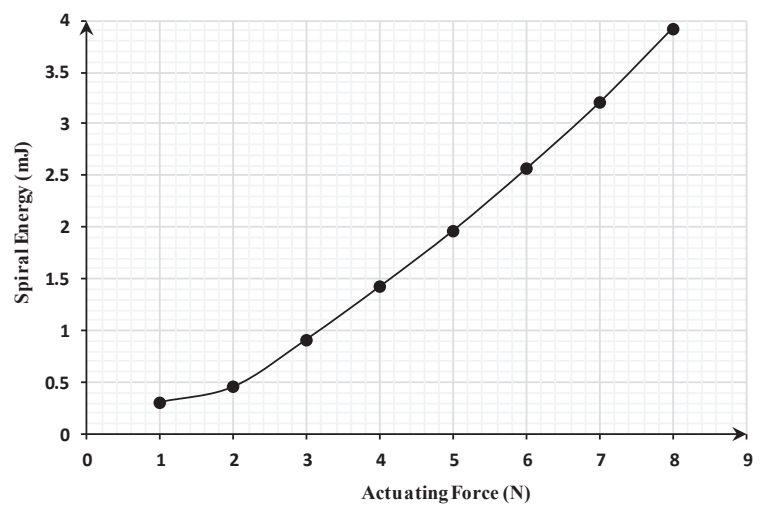

Fig. 13. Spiral energy.

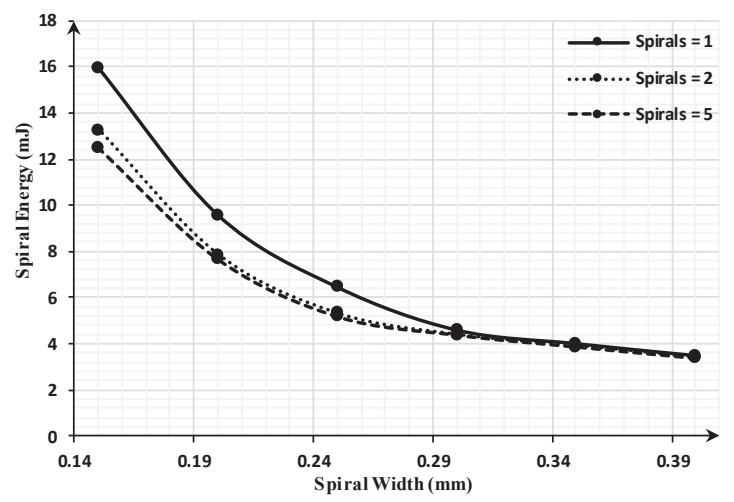

Fig. 14. Effects of width and spiral number on storage energy.

Figure 14 shows that the spiral width is the most important parameter of the proposed energy-harvesting cell. By decreasing the width, the storage could rotate further and will have 
a larger rotation angle, and thus more stored energy. The number of spirals is also important; increasing the number of spirals causes less rotation and thus less stored energy.

\subsection{Multistep analysis}

Multistep analysis of the proposed structure has also been performed. To model the force produced by the heart vein as a function of time, a sequence of pulsed forces has been considered as depicted in Fig. 15. For a beat rate of 70 times per min, a pulse sequence of 0.85 $\mathrm{s}$ period is required. It could be a good approximation to take the period as $1 \mathrm{~s}$ to simplify the implementation. The pulses have $0.1 \mathrm{~s}$ duration and $7 \mathrm{~N}$ of amplitude, as shown in Fig. 15.

The elastic strain energy density in multistep mode of operation for a cell with 5 turns, 0.7 $\mathrm{mm}$ thickness, $0.15 \mathrm{~mm}$ spiral width, and 58 teeth is shown in Fig. 16. Figure 16 shows that the cell could store mechanical energy in the form of mechanical stress. The maximum stress curve of the cell also validates the cell harvesting and storing behavior. As shown in Fig. 16, by delivering each pulse, the cell stress was increased, which leads to increased stored energy. The elastic strain energy density (strain energy per unit volume) in multistep mode of operation for a cell with 2 turns, $1 \mathrm{~mm}$ of spiral width, and 78 teeth is illustrated in Fig. 17.

The parabolic increase in energy against a linear one in stress is worth noting. This can be understood by the fact that energy is proportional to the squared stress. Simulated results show that harvesting and storing mechanical force in bioapplication could be carried out.

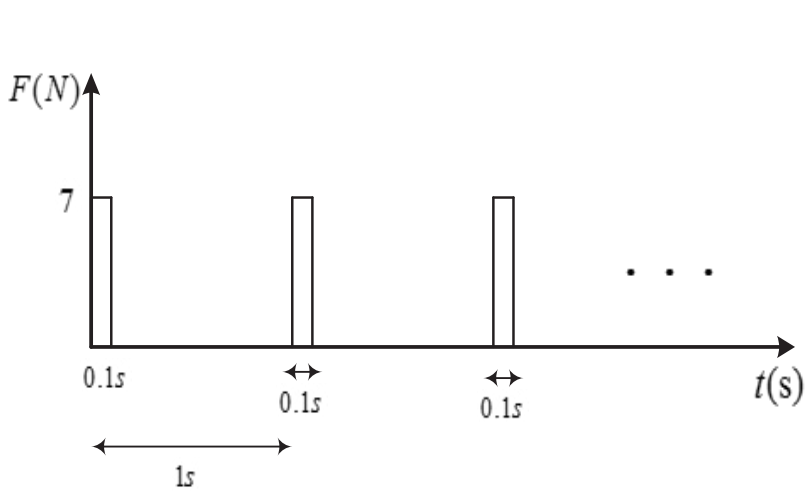

Fig. 15. Dynamic model of mechanical actuating force for heartbeat.

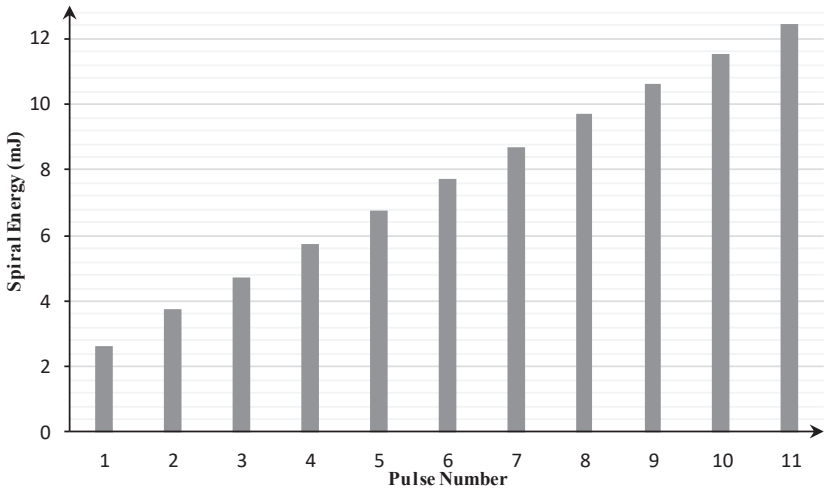

Fig. 16. Multistep energy storage in the cell.

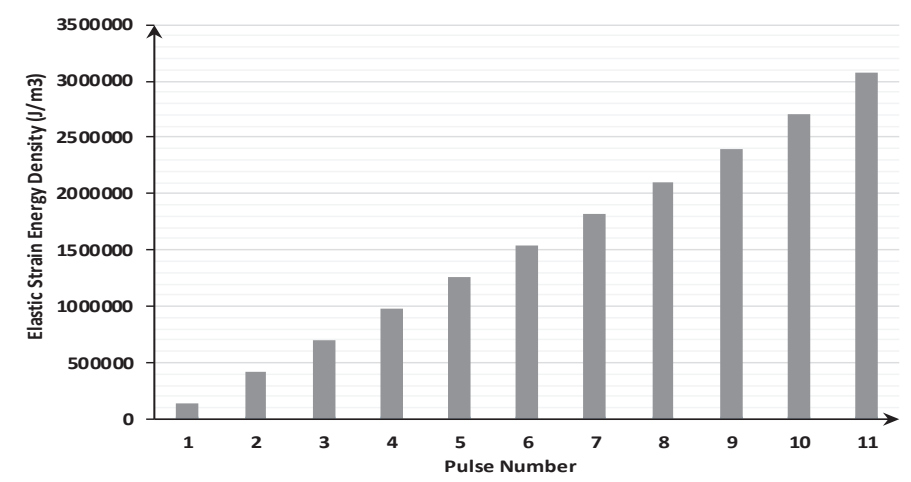

Fig. 17. Elastic strain energy density. 


\section{Conclusions}

A new mechanical EH was proposed. Three different parts formed the structure, namely, the mechanical actuator, storage, and latch. The structure was assessed both in theory and through simulations. For a typical cell, the total area of the structure was $30.06 \mathrm{~mm}^{2}$ while the thickness was considered as $0.7 \mathrm{~mm}$. Different equivalent cells were extracted according to thickness, the number of spiral turns, spiral ring width, and the number of teeth. The maximum angle of rotation was $36^{\circ}$ and the minimum angle of rotation was $6.2^{\circ}$. Heart beating was modeled by a $7 \mathrm{~N}$ force, and the cell was actuated using a heartbeat model. By applying a $7 \mathrm{~N}$ mechanical force to the actuator, mechanical energy of up to $3.21 \mathrm{~mJ}$ was stored per unit volume in the storage. To model heartbeat vibration, multistep analysis of the structure was performed. The results showed that the proposed mechanical EH is a convenient option for biomedical energy harvesting applications.

\section{References}

1 C. B. Williams and R. B. Yates: Sens Actuators, A 52 (1996) 8.

2 C. B. Williams, C. Shearwood, M. A. Harradine, P. H. Meller, T. S. Birch, and R. B. Yates: IEE Proc. Circuits Devices Syst. 148 (2001) 337.

3 T. J. Kazmierski and S. Beeby: Energy Harvesting Systems: Principles, Modeling and Applications (Springer, New York, 2011).

4 N. Mohammad Pour: Dr. Thesis, Departments of Mechanical and Aerospace Engineering, Politecnico di Torino, Torino (2014).

5 P. Glynne-Jones, S. Beeby, and N. White: IEE Proc. Sci. Meas. Technol. 148 (2001) 68.

6 S. P. Beeby, M. J. Tudor, and N. White: Meas. Sci. Technol. 17 (2006) 175.

7 H. A. Sodano, D. J. Inman, and G. Park: Shock Vib. Dig. 36 (2004) 197.

8 S. J. Roundy: Dr. Thesis, University of California, Berkeley (2003).

9 R. Amirtharajah and A. P. Chandrakasan: IEEE J. Solid-State Circuits 33 (1998) 687.

10 L. Parkhouse: U.S. Patent, No. 7003353 (2006).

11 K. Murakawa, M. Kobayashi, O. Nakamura, and S. Kawata: IEEE Eng. Med. Biol. Mag. 18 (1999) 70.

12 T. Torfs, V. Leonov, and R. Vullers: Sens. Transducers J. 80 (2007) 1230.

13 A. Yadav, K. Pipe, and M. Shtein: J. Power Source 175 (2008) 909.

14 T. Huesgen, P. Woias, and N. Kockmann: Sens. Actuators, A 145 (2008) 423.

15 X. Wang, J. Liu, J. Song, and Z. Wang: Nano Lett. 7 (2007) 2475.

16 M. J. Ramsay and W. W. Clark: Proc. 2001 SPIE Smart Structures and Materials: Industrial and Commercial Applications of Smart Structures Technologies (SPIE, 2001) 429.

17 J. W. Sohn, S. B. Choi, and D. Y. Lee: J. Mech. Eng. Sci. 219 (2005) 429.

18 S. R. Platt, S. Farritor, K. Garvin, and H. Haider: IEEE ASME Trans. Mechatron. 10 (2005) 455.

19 J. Paulo and P. Gaspar: Proc. 2010 World Cong. Engineering (WCE, 2010) 168.

20 L. C. Rome, L. Flynn, E. M. Goldman, and T. D. Yoo: Science 309 (2005) 1725.

21 S. Roundy, P. K. Wright, and J. Rabaey: Comput. Commun. 26 (2003) 1131.

22 D. Zhu, M. J. Tudor, and S. P. Beeby: Meas. Sci. Technol. 21 (2009) 022001.

23 S. Roundy and P. K. Wrigth: Smart Mater. Struct. 13 (2004) 1131.

24 G. T. Hwang, M. Byun, C. K. Jeong, and K. J. Lee: Adv. Health Mater. 4 (2015) 646.

25 D. Fan, Y. Liu, F. Han, and J. Dong: Sens. Actuators, A 187 (2012) 190.

26 P. D. Mitchelson, P. Miao, B. H. Stark, E. M. Yeatman, A. S. Holmes, and T. C. Green: Sens Actuators, A 115 (2004) 523.

27 M. El-hami, P. Glynne-Jones, N. M. White, and S. Beeby: Sens. Actuators, A 92 (2001) 335.

28 B. C. Lee and G. S. Chung: Trans. Electr. Electron. Mater. 14 (2013) 143.

29 C. Dagdeviren, B. D. Yang, Y. Su, P. L. Tran, P. Joe, E. Anderson, J. Xia, V. Doraiswamy, B. Dehdashti, X. Feng, B. Lu, R. Poston, Z. Khalpey, R. Ghaffari, Y. Huang, M. J. Slepian, and J. A. Rogers: Proc. Natl. Acad. Sci. U.S.A. 111 (2013) 1927.

30 A. M. Howatson, P. G. Lund, and J. D. Todd: Engineering Tables and Data (Chapman and Hall, London, 1972) p. 41. 